\title{
It's not over till it's over: a narrative review of Long COVID
}

\author{
Joseph V. Pergolizzi ${ }^{1}$, Jo Ann LeQuang ${ }^{1, \star}$, Peter Magnusson ${ }^{2,3}$, Dariusz Myrcik ${ }^{4,5}$, \\ Giustino Varrassi ${ }^{5}$
}

${ }^{1}$ NEMA Research Group, Naples, FL 34108-1852, USA

${ }^{2}$ Centre for Research and Development, Uppsala University/Region Gävleborg, 80188 Gävle, Sweden

${ }^{3}$ Cardiology Research Unit, Department of Medicine, Solna, Karolinska Institutet, 17177 Stockholm, Sweden

${ }^{4}$ Department of Internal Medicine, University of Silesia, 41813 Katoviche, Poland

${ }^{5}$ Paolo Procacci Foundation, Via Tacito 7, 00193 Rome, Italy

\section{*Correspondence}

joannlequang@gmail.com

(Jo Ann LeQuang)

\begin{abstract}
Viral persistence following acute COVID infection is increasingly being reported by patients and gradually being recognized as a medical syndrome. Like much about COVID, this so-called Long COVID is perplexing. It is associated with numerous symptoms, foremost among them profound fatigue, and often occurs in a relapsing/remitting pattern. There is one "living" guideline for managing Long COVID and even terminology and definitions of the syndrome are in flux. Long COVID occurs in patients who have recovered from the acute infection and this may be viral persistence, a form of autoimmunity, or the long-term results of organ damage sustained during the acute infection. Symptoms have been reported up to six months after acute infection with no clear association between the severity of the acute infection and the presence or absence of Long COVID. The symptoms of the acute illness do not necessarily align with the symptoms of Long COVID. Disruptions to the autonomic function in Long COVID are particularly puzzling, including orthostatic intolerance syndrome (which may not have occurred during the acute infection). Loss of the sense of smell and taste is one symptom that appears common in both acute and Long COVID; on the other hand, fever is more prevalent in acute than Long COVID. Research is urgently needed to better understand Long COVID, for example: what is the role of elevated biomarkers such as D-dimer and C-reactive protein in Long COVID? Is Long COVID one or more than one syndrome? How can patients with Long COVID be appropriately treated?
\end{abstract}

\section{Keywords}

COVID-19; COVID therapy; Fatigue; Immunobiography; Long COVID; Long-haul COVID; Oxidative stress; Therapy

\section{Introduction}

Viral persistence is a well-known phenomenon and has been observed in the relapsing/remitting symptoms of the Chikungunya virus or the unrelenting and prolonged symptoms of Ebola infection [1,2]. Three main concepts explain viral persistence: the virus sequestering in immuneprivileged sites, aberrant immune response, and autoimmunity [3]. The emergence of persistent symptoms of the SARS-CoV2 infection, sometimes called "Long-haul COVID-19" or just "Long COVID" is emerging as a secondary public health crisis in the wake of the pandemic. Like other postviral syndromes, Long COVID is frequently associated with chronic fatigue and other conditions ranging from mild to debilitating [4].

Little is scientifically demonstrated about Long COVID other than the fact that certain symptoms seem to persist in a subset of those who survive acute COVID infection; these symptoms may be the same, similar, or different from the acute symptoms. Because of the novelty of this syndrome, the evidence for Long COVID to date comes mainly in the form of anecdotal reports and there is a paucity of solid research on this condition [5]. Modern academic medicine values rigorous clinical trials with results published in reputable peer-reviewed journals by notable authorities above patient anecdotes shared on social media, but the number of patients speaking out about extended or unusual symptoms after acute infection could not be long overlooked [6]. In fact, the first account of the main 50 symptoms associated with Long COVID came not from physicians or scientists at all, but rather from a social media community of acute COVID survivors who were self-reporting their experiences $[7,8]$. This is an interesting novelty emerging from the COVID pandemic and differentiating it from the previous pandemic of the last century [9].

Long COVID poses formidable challenges to our already beleaguered healthcare system, because a subset of those infected with COVID may experience symptoms after viral clearance and the course and consequences of these symptoms is not known. The goal of this study was to explore what is currently known about Long COVID, what is speculated based on good evidence, and what is not yet known. This is a narrative rather than systematic review; there are few rigorous studies on Long COVID. Indeed, in the summer of 2020, complaints 
from COVID survivors about persistent symptoms following a negative COVID test were sometimes minimized or even frankly disbelieved by the healthcare profession [10], perhaps because clinicians were overwhelmed with caring for acute patients.

There remains to date no universally recognized definition of Long COVID [11]. The absence of a consensus definition can lead to clinical confusion, which in turn can contribute to the marginalization of patients, and thwart attempts to better diagnose and treat Long COVID [12]. SARS-CoV-2 is a novel zoonotic virus and, as such, persistent symptoms following the acute infection might more traditionally be called "chronic COVID syndrome" [13]. Nevertheless, the term Long COVID, largely introduced by laypeople, has found acceptance in scientific and medical databases.

\section{Methods}

The authors searched the literature for keywords "Long COVID", "Long-haul COVID", in January and February 2021 and evaluated that body of literature and its references. We included articles that were peer-reviewed, written in English, and described Long COVID. We excluded patient-written content. Papers were evaluated by the authors and those with a primary focus on the specific phenomenon of postviral COVID symptoms (Long COVID) were included. In some cases, definitive websites were searched as well and the bibliographies of articles consulted. For this publication, the syndrome will be called "Long COVID" although the terms Long-haul COVID, Long COVID-19, and chronic COVID are used in the literature and some sources use the lowercase, that is, Long COVID.

\section{Results}

There were 69 results for Long COVID and 12 for Longhaul COVID with some overlap. We found that terminology remains to be better defined, that a long list of symptoms has been explored as being associated with Long COVID, and its epidemiology is still being defined. In fact, it may be that there is more than one syndrome being called Long COVID and immunobiography may be an important part of better understanding the inherent plasticity of the immune system with respect to viruses and other pathogens. It appears that certain special populations may be more vulnerable to Long COVID than others and guidelines are starting to address Long COVID. Finally, the clinical considerations with respect to Long COVID are presented. The body of literature on Long COVID is not large but it is growing. Presented here is what is currently known, what is suspected, and what remains unknown.

\subsection{Terminology}

Terminology is further challenged by the fact that symptoms of the persistent condition vary widely among patients, with some reporting anxiety and brain fog, others respiratory conditions, and still others persistent cardiovascular conditions. Many patients report multiple symptoms, which may not always match their acute symptoms. In cases of other postviral syndromes, for example viral hepatitis, the transition from the initial acute infection to the more prolonged symptoms are generally described as "acute" and "chronic" [13]. A temporal cutoff has been proposed to separate acute from chronic COVID at three weeks, based on observational data [13]. Other authors have called this syndrome that occurs three weeks after acute infection "post-acute COVID" or "post-COVID syndrome", which then transitions to "chronic COVID" at 12 weeks [14, 15]. For others, Long COVID is defined to occur in those who have any COVID symptoms $>28$ days post-diagnosis of the initial infection [11, 15]. The Centers for Disease Control and Prevention (CDC) have proposed staging COVID infection into three phases, with the acute phase defined by the period of active viral replication, a post-acute hyperinflammatory phase (defined as a positive antibody test two weeks after diagnosis), and late sequelae or Long COVID after four weeks [16]. See Table 1 (Ref. [16, 17]). The consumer press and social media favor the patient-coined term Long COVID without differentiating the three, four, or 12 week markers [14]. Since our understanding of Long COVID is in its infancy and the syndrome continues to surprise epidemiologists, it is perhaps premature to arrive at hard definitions.

\subsection{Epidemiology of Long COVID}

For better or worse, social media has played and continues to play a major role in the COVID pandemic, including in the recognition of Long COVID as a genuine medical syndrome [18]. Patient support groups such as WeAreBodyPolitic . com and the Long COVID group on Facebook provided the first patient self-reports of Long COVID and allowed these individuals to share their stories outside the scrutiny of the medical establishment. At the time of publication, Long COVID has been discussed more on social media among patients and their families than in the medical literature [7]. This does not mean such social media reports should be discounted or reduced to mere "anecdotal" stories from random patients. Indeed, many descriptions of Long COVID on social media come from healthcare professionals who became infected on the job and have the medical expertise to describe their symptoms accurately [7]. A Symptom Study app was developed in the United Kingdom to crowd-source data about COVID-related experiences directly from patients; this app became a source of information about Long COVID as well. Information obtained from almost 4 million individuals found that about $10 \%$ of COVID patients experience symptoms that last three weeks or longer [5]. Other studies have found the rate of persistent symptoms to occur in much larger proportions of those who have had acute COVID infections [19-23].

While men are at elevated risk for contracting the SARSCoV-2 infection, women appear to be at greater risk of developing Long COVID in the aftermath of the acute infection [24]. Patients with comorbidities, particularly obesity (body mass index $\geq 30$ ) and/or psychiatric disorders, are more likely to have prolonged symptoms of COVID disease [17, 20]. There have been no conclusive studies that can associate specific symptoms of acute COVID with persistent symptoms of Long COVID [25], nor can the severity of the acute infection be 
TA B L E 1. The stages of infection with the SARS-CoV-2 virus as defined by the Centers for Disease Control and Prevention (CDC) [16, 17]. Note that fatigue, a symptom of Long COVID frequently reported by patients, does not appear on this guidance from the CDC.

$\begin{array}{lccc} & \text { Acute COVID } & \text { Hyper inflammatory stage } & \text { Long COVID } \\ \text { Description } & \begin{array}{c}\text { Active viral replication } \\ \text { Initial host response }\end{array} & \text { Dysregulated host response } & \begin{array}{c}\text { Uncertain, pathophysiological } \\ \text { pathways may be involved }\end{array} \\ \text { Duration } & <2 \text { weeks } & 2-4 \text { weeks } & >4 \text { weeks } \\ \text { Pathology } & \text { Viral replication } & \text { Immune response } & \text { Unknown } \\ \text { Primary symptoms } & \text { Fever, dry cough, dyspnea, } & \begin{array}{c}\text { Gastrointestinal, cardiovascular, } \\ \text { respiratory, neurological, } \\ \text { myalgia, anosmia, nausea, diarrhea }\end{array} & \begin{array}{c}\text { Cardiovascular, pulmonary, } \\ \text { neurological, psychological }\end{array} \\ \text { Diagnostic tools } & \text { Viral test (PCR) } & \text { Antigen testing } & \text { None }\end{array}$

$P C R$, polymerase chain reaction.

associated with the likelihood of developing Long COVID [26].

\subsection{Symptoms of Long COVID}

Long COVID may be described as persistent symptoms in patients who have recovered from acute SARS-CoV-2 infection, with the most frequently reported symptoms fatigue, exercise intolerance, brain fog, cognitive deficits, disordered sleep patterns, and a range of autonomic symptoms, such as night sweats, gastroparesis, gastrointestinal disturbances, and peripheral vasoconstriction [7]. This list is not exhaustive; other frequently reported symptoms include dyspnea, joint pain, chest pain, cough, and anosmia [19]. While chills and fever associated with acute SARS-CoV-2 infection tend to resolve with viral clearance, symptoms of fatigue and cough were more likely to persist [20]. More symptoms of Long COVID have also been described: myocarditis, pericarditis, microvascular angina, cardiac arrhythmias including atrial flutter, atrial fibrillation, and ventricular tachycardia, dysautonomia (postural orthostatic tachycardic syndrome), mast cell activation syndrome, interstitial lung disease, thromboembolic conditions, myelopathy, neuropathy, neurodegenerative disorders, renal impairment, hepatitis, abnormalities in liver enzymes, and dystonia [3]. Loss of the sense of smell and/or taste is commonly reported as a symptom of both acute and Long COVID [22, 27]. In addition, symptoms such as new-onset diabetes, new-onset hypertension, new-onset thyroiditis, newonset allergies, and new-onset anaphylaxis have been reported in the setting of acute infection, but it is not clear if these symptoms persist after the acute infection resolves to become part of the Long COVID syndrome [3]. Symptoms persisting after acute infection are frequently reported and may be present for an extended period of time, see Table 2 (Ref. [19-23, 28]).

Certain symptoms are puzzling. For example, disruptions in autonomic function have been reported subsequent to acute COVID infection that result in orthostatic intolerance syndrome. It is not clear if this condition relates to a virusmediated attack or an attack mediated by the immune system in the wake of COVID infection [14]. Indeed, symptoms such as these that may occur subsequent to acute infection may even be unrelated to the virus. Autonomic symptoms, such as heart palpitations, tachycardia, dyspnea, syncope, and so on have been reported among a case series of six patients in early 2020 who were suspected to have had acute COVID (one case was confirmed, five others has suspected COVID with symptoms compatible with the disease) [14]. These early data were gathered at a time when COVID testing was not widely available. The six patients in this case series were all females between the ages of 26 and 50 and all presented with orthostatic intolerance with resting or postural hypotension and/or tachycardia [14]. In a person with any sort of viral infection, hypovolemia may occur, which can be further exacerbated by prolonged bed rest and subsequent deconditioning. This hypovolemic state can diminish cardiac output, reduce stroke volume, interfere with the baroreflexes, and reduce the body's neurological sympathetic response [14].

The loss of the sense of smell is reported often in both acute and Long COVID. It is intriguing that the anosmia associated with acute COVID infection is usually not accompanied by rhinitis [27, 29]. ACE2 receptors, the entry point for the SARS-CoV-2 virus, are densely expressed in the nasal mucosa, and anosmia was likewise common in patients suffering from the Severe Acute Respiratory Syndrome (SARS) epidemic more than a decade ago [30]. Initially, it had been suspected that anosmia was indicative of central nervous system involvement, but two factors point against this. First, most acute COVID patients who experience anosmia recover their sense of smell within a week or two after infection [31]. Second, central nervous system symptoms occur in far fewer patients than anosmia [32], suggesting that they are unrelated. The virus may instead target non-neuronal cells that express ACE2 receptors, which would include the olfactory epithelium sustentacular cells, microvillar cells, Bowman's gland cells, horizontal basal cells, and olfactory bulb pericytes, among others [33]. The persistence of anosmia in some Long COVID patients may be the result of slow recovery of these nonneuronal cells.

Dermatological manifestations of COVID may occur in the acute phase and can persist, but are less well described in the literature. A case report of acute vasculitis in an 11-year-old COVID patient reported erythematous lesions on the feet and ulcerative lesions with dyschromia of the nails [34]. Other cutaneous symptoms may include pernio, morbilliform, urticaria, 
TA B L E 2. Studies following patients who survived acute SARS-CoV-2 infection and were interviewed about symptoms show that persistent symptoms are pervasive and long lasting [19-23, 28].

\begin{tabular}{|c|c|c|c|c|c|}
\hline Study & $\begin{array}{l}\text { Time elapsed since } \\
\text { acute infection }\end{array}$ & Fatigue & Cough & Dyspnea & Other \\
\hline $\begin{array}{l}\text { Carfi } 2020 \\
143 \text { hospitalized pt in Italy }\end{array}$ & Mean 60 days & $53 \%$ & $15 \%$ & $43 \%$ & $\begin{array}{c}55 \% \text { had } \geq 3 \\
\text { symptoms and } 44 \% \\
\text { had reduced QoL }\end{array}$ \\
\hline
\end{tabular}

Huang 2021

1733 hospitalized COVID pt in China

Median 186 days

fatigue and/or
muscle

weakness

Mandal 2020

384 hospitalized pt in UK

Moreno-Perez 2021

277 patients with acute COVID in Spain
Median 54 days

Mean 77 days

$$
69 \%
$$

$35 \%$

NR

NR

$26 \%$ had problems sleeping and $23 \%$ had depression or anxiety

$15 \%$ depression

$51 \%$ still had symptoms; $18 \%$ had headaches

Petersen 2020

180 adults with COVID in Faroe Islands, Mean 125 days Denmark

Tenforde 2020

CDC Phone interview of 292 adults with Median 16 days positive COVID test in the United States post-test

$24 \%$ of those $\quad \mathrm{NR}$
who were
symptomatic
in acute phase

\begin{tabular}{|c|c|c|c|}
\hline $\begin{array}{l}\text { (Of those } \\
\text { with } \\
\text { symptoms) }\end{array}$ & $\begin{array}{l}\text { (Of those } \\
\text { with } \\
\text { symptoms) }\end{array}$ & $\begin{array}{l}\text { (Of those } \\
\text { with } \\
\text { symptoms) }\end{array}$ & $\begin{array}{l}35 \% \text { had not } \\
\text { recovered at time of } \\
\text { phone interview }\end{array}$ \\
\hline $35 \%$ & $43 \%$ & $29 \%$ & \\
\hline
\end{tabular}

$$
\text { NR } \quad \begin{gathered}
53 \% \text { still had at } \\
\text { symptoms }
\end{gathered}
$$

NR, not reported; Pt, patients; QoL, quality of life.

papulo-squamous eruptions and chilblains [34]. A study of 163 acute COVID patients with dermatological symptoms reported that $6.8 \%$ of acute COVID patients with pernio ("COVID toes") had pernio that lasted more than 60 days [35].

While fever is a hallmark symptom of acute COVID infection, it seems to be rare in Long COVID [7]. However, most other symptoms of acute COVID can also occur in Long COVID. Long COVID often involves multiple symptoms; a survey of 274 COVID patients reporting persisting symptoms, found the median number of lingering symptoms was seven [20].

Biomarkers for Long COVID have not yet been extensively investigated. In a study of 384 hospitalized COVID Patients in the United Kingdom followed for a median of 54 days post-discharge, some patients exhibited elevated biomarkers. Among the COVID patients who had abnormally elevated D-dimer and C-reactive protein levels at hospital discharge, $30.1 \%$ and $9.5 \%$, respectively, continued to have these elevated levels at follow-up four to six weeks post-discharge [28]. A single-center study from London reported that $30 \%$ of 229 patients hospitalized with acute COVID had elevated D-dimer levels a median of 54 days after hospital discharge [28]. The D-dimer score at hospital admission was recorded for 176 COVID patients and had a median value of 785 and an interquartile range of 510 to 1486 . At predischarge, this value was 878.5 (547.4-2522.5) and at follow-up four to six weeks later, it was 384 (242-665). The meaning of these
D-dimer levels is not clear [28]. Upon hospital admission, $87 \%$ of this same cohort had a chest X-ray, of which $15 \%$ were normal, $29 \%$ indeterminate, and $56 \%$ typical of COVID patients. About two-thirds of patients received a chest X-ray at follow-up, of which $62 \%$ were considered normal, $27 \%$ showed improvement, $2 \%$ were unchanged, and $9 \%$ showed significant deterioration. It is interesting, that of the patients with a chest $X$-ray showing marked deterioration $(n=23), 9 \%$ had had normal X-rays at admission, $48 \%$ had indeterminate radiographic findings, and $43 \%$ had "typical COVID" chest $\mathrm{X}$-rays [28]. It is worth noting that more than half of those with normal or indeterminate chest radiographs during acute COVID showed deterioration at follow-up, suggesting that Long COVID may be more dangerous than just lingering mild symptoms.

Mental symptoms have also been reported. A frequent complaint among Long COVID patients is brain fog, confusion, changes in cognition, and loss of memory [36]. Patients also reported emotional distress with Long COVID, manifesting as fearfulness, worry about their health, concern that they might never recover, all of which could be exacerbated by lack of consistent Long COVID care or dismissal of their symptoms by healthcare professionals and family [36]. Anxiety and depression are frequently reported with Long COVID but it is not always clear if Long COVID sequelae, new-onset conditions, or if the patient had pre-existing mental health disorders or risk factors for psychological distress. After 
all, COVID infection is a distressing condition with many unknowns and living in pandemic conditions for months on end has been emotionally challenging for everyone, especially for healthcare professionals and others on the frontlines of care [37]. The sometimes-promoted view of COVID as a dichotomous condition, that is, either one has it or one has recovered, can further promote frustration and even anxiety among those trying to navigate this novel syndrome [36].

\subsection{Is Long COVID more than one syndrome?}

Long COVID sometimes occurs as a prolonged illness where acute infection transitions seamlessly to a long period, in which a subset of the initial symptoms persist. For other patients, Long COVID is a relapsing/remitting condition where patients initially recover from acute illness, then report cyclic recurrence of certain symptoms [7]. This distinction in natural course may be important to understanding Long COVID. For instance, it has been observed that patients who suffer from moderate to severe or very severe acute COVID infections may exhibit Long COVID in the form of prolonged symptoms, while those who have mild cases of the acute infection are more likely to develop the relapsing/remitting form of Long COVID [16, 38]. Thus, Long COVID may exhibit as many as three distinct and unrelated manifestations: persistent residual symptoms of the acute infection, symptoms related to organ dysfunction caused by the acute infection, and new symptoms [26].

Patients with severe cases of COVID treated in intensive care units (ICUs) may experience post-ICU syndrome- not unique to COVID - but which may be confused with Long COVID [17]. Patients with post-ICU syndrome may experience cognitive impairment, memory loss, shorter attention span, deterioration of executive function, muscle weakness or atrophy, joint contractures, diaphragmatic dysfunction, laryngeal injury, dysphagia, dysphonia, anxiety, and depression [39]. COVID patients in the ICU are exposed to conditions that may exacerbate post-ICU syndrome, for example, they may be isolated; have little to no contact with family and friends; have less than usual access to clinicians and rehabilitation services due to hospital staffing constraints during the pandemic; have been sedated or intubated; and lack adequate information about this new and potentially fatal disease [40]. Post-ICU syndrome is persistent. In a study of 196 patients who had been hospitalized in an ICU unit for acute respiratory distress syndrome, at a median of 33 to 39 months after their ICU stay, $52 \%$ reported continuous or recurring symptoms, $38 \%$ had prolonged anxiety, $32 \%$ had depression, and $23 \%$ had symptoms akin to posttraumatic stress disorder [41, 42].

Persistent symptoms following viral infection are not unique to COVID. For example, the Epstein-Barr and Guillain-Barré viruses are associated with chronic symptoms [43] and the mosquito-borne Chikungunya virus is likewise associated with periodic recurrences of symptoms over months following the initial illness [44]. Patients with SARS, a coronavirus disease caused by a virus similar to the SARS-CoV-2, have also reported long-term symptoms that include fatigue, weakness, musculoskeletal pain, depression, and poor sleep quality, in a condition named Chronic Post-SARS Syndrome [45].
Organ damage may occur in acute COVID infections that persists after viral clearance, including damage to the pulmonary, cardiovascular, thromboembolic, neurological, and renal systems [26]. While cardiac symptoms of acute COVID infection are not frequently reported, a study of 100 COVID patients using magnetic resonance imaging reported that $78 \%$ of those who had recovered from the acute infection still had some degree of cardiac involvement in the form of myocardial inflammation, regional fibrosis, or pericardial enhancement; in this study $67 \%$ of patients had no or only mild cardiac symptoms during acute illness [46]. Further study is warranted to determine whether those who have cardiac dysfunction following acute COVID infection have pre-existing cardiac conditions or risk factors for cardiovascular disease.

Acute COVID infection is associated with an elevated risk for venous thromboembolism (VTE) [47] but the risk of VTE in patients who recover from COVID is not known [48]. VTE is not typically listed among the Long COVID symptoms but should be considered in future studies.

Viral reinfection cannot be ruled out as a potential occurrence, particularly in patients with suppressed immune systems [17]. A case study reports that a 57-year-old man with a confirmed but asymptomatic case of COVID developed a mild but symptomatic case of COVID about three months later. It is not clear whether this was reinfection from a communitybased source, viral reinfection, or a delayed immune response [49]. Cases of potential reinfection are rare but have been reported; there is no strong evidence with respect to whether a recovered COVID patient can become reinfected. In this context, it is important to note that replication-competent virus is rarely found in recovered COVID patients after 20 days on onset of acute symptoms [50]. On the other hand, it has been proposed that the virus may be able to sequester itself in socalled "immunologically privileged locations", from which it is difficult for the body to remove the virus [26].

Fatigue is among the most frequently reported Long COVID symptoms, but fatigue is a diffuse symptom with multiple potential etiologies, including post viral fatigue, which may occur after any number of viral infections and is not unique to COVID [17, 51]. Myalgic encephalomyelitis (ME) and chronic fatigue syndrome (CFS) are well-known syndromes with an obscure etiology; many patients diagnosed with ME/CFS had a viral infection prior to onset of symptoms but this was not necessarily a COVID infection [7]. ME/CFS includes symptoms of physical exhaustion, poor sleep, depressed mood, memory deficits and "accelerated aging" [17]. The fatigue may also be related to the EpsteinBarré virus, a herpes virus which is associated with relapsing/remitting patterns that seems to fit Long COVID symptomology [17]. It is not clear if ME/CFS is a disease or a constellation of symptoms, but it has transitioned from being viewed as a biopsychosocial phenomenon to being more widely recognized as a form of chronic illness [52]. The symptoms of ME/CFS and Long COVID overlap in many cases, and it is not clear if they may be the same condition in some patients or unrelated conditions despite their symptomatic similarities. 


\subsection{The role of immunobiography}

Immunobiography describes a patient's personal history of pathogenic exposures and the presence of antigens, which can influence that patient's inflammatory response to other stimuli $[53,54]$. The plasticity of the immune system preserves an individual record or biography of the individual's experiences and continuous adaptation to pathogens. Thus, immunobiography can help explain the genetic contributions to differences in immune systems and may explain sex-based differences as well $[53,55]$. Since the immune system undergoes remodeling with advancing age, the aging body becomes more and more vulnerable to infections, malignancies, and autoimmune disorders [56]. For example, over time, the body accumulates material produced by living organisms, including cellular debris, metabolites, molecular aggregates from incomplete degradation of non-enzymatic reactions, and a variety of other products known as "molecular garbage", all of which reduce the body's ability to fight off new pathogens [57]. It is not known if and to what extent immunobiography can help differentiate those patients with acute COVID infection who will go on to develop Long COVID versus those who will fully recover in a short period of time.

\subsection{Special populations and Long COVID}

The term inflammaging (inflammation + aging) refers to chronic subclinical systemic inflammation associated with aging and can be assessed with specific biomarkers, such as Creactive protein, interleukin (IL)-6, IL-18, and tumor necrosis factor $[54,55]$. Inflammaging may be described as the chronic condition that underlies the aging process [53]. The concept of inflammaging may have profound ramifications for Long COVID among geriatric COVID survivors, who may be at elevated risk for morbidity, disability, frailty, and other infections [55].

Chronic inflammation has been linked to many chronic conditions including metabolic syndrome, type 2 diabetes mellitus, non-alcoholic fatty liver disease, cardiovascular diseases, cancer, autoimmune disorders, neurodegenerative conditions, and others [58]. With aging, a more complex, multisystem, chronic inflammatory state may occur and factors such as unhealthful diet, obesity, comorbidities, sedentary lifestyle, stress, social isolation imposed by the pandemic, disordered sleep, and mental health problems may exacerbate this agerelated condition [58].

Cellular senescence may contribute to the pathogenesis of sustained chronic inflammation. Cellular senescence may occur for any number of reasons: genomic instability, metabolic disorders, oxidative stress, energy crises, and others [54, 59]. Cellular senescence stops normal cell proliferation, resists apoptosis, and can cause the massive release of senescenceassociated secretory phenotype (SASP) molecules, including various cytokines, chemokines, and other molecular species [55]. Because the SARS-CoV-2 may activate a powerful inflammatory response, it has been speculated that this "cytokine storm" may cause an immune system paralysis of sorts, starting with a hyperinflammatory phase followed by the compensatory anti-inflammatory response, leading to immunosuppression and increasing the patient's vulnerability to secondary infections and immunosenescence [55]. With immune system paralysis, a patient may be in a hyperinflammatory state yet lose the ability to react to a new inflammatory insult [55]. Cellular senescence of various tissue systems may launch a vicious cycle where senescence results in SASP-related proinflammatory factors leading to inflammation and triggering more cellular senescence [55].

The trajectory of acute COVID seems to differ markedly among pediatric, young adult, and geriatric patients with children far less likely to experience severe symptoms than adults and seniors. However, just as with adult patients, Long COVID may occur in healthy children who do not experience severe symptoms with acute COVID [60]. A case report described five pediatric patients $(80 \%$ female) between the ages of 9 and 15 years (median 12) with diagnosed COVID infections who had COVID symptoms that persisted between six and eight months [60]. Two months after the initial onset of acute COVID, all five children reported symptoms of fatigue, dyspnea, chest pain, and palpitations. Three of the children reported abdominal pain, memory loss, depression, cutaneous symptoms, and myalgia. Two children had remitting fever, problems sleeping, joint pain, diarrhea, and vomiting. One child had problems with taste and smell for two months after onset of acute COVID as well as poor appetite, chronic coughing, and numbness. Four of the five children suffered symptoms every day while one of the five had intermittent symptoms characterized by "good days and bad days" [60]. Thus, Long COVID may affect pediatric patients.

Inequities in healthcare have been a hallmark of COVID infections in many parts of the world and such disparities may become evident with the emergence of Long COVID syndrome. In addition to disadvantaged communities of minority populations and those of low socioeconomic status, it may be that individuals with mental health disorders, learning disabilities, or problems communicating (language barrier, dementia) face unequal care and treatment [15].

\section{Clinical implications of Long COVID}

Long COVID is a perplexing and challenging condition and patients who experience this syndrome often feel misunderstood, marginalized, and dismissed by the healthcare system. Even when clinicians show support for these patients, there is limited understanding of Long COVID, its natural course, and appropriate therapies. While the public may have a reasonable understanding of acute COVID, Long COVID is fraught with so many unknowns that it can be extremely distressing to patients trying to understand what is happening to them. For clinicians, it is important to acknowledge the symptoms and experiences of patients who suspect they have Long COVID, and healthcare professionals may better avoid providing hasty answers or dismissing their concerns entirely [36]. Indeed, many patients report feeling stigmatized by family and the medical profession for reporting Long COVID symptoms and not simply "getting over" what should be a relatively mild illness [61]. Since Long COVID is not experienced by all COVID survivors, it is easy to think that the Long COVID patients are exaggerating their complaints. While these patients may require both emotional support and validation, on 
the one hand, they also should get symptomatic treatment, on the other hand [36]. Continuity of care and standards of care are important and must be established rapidly, as patients at this time with similar histories and symptoms may be treated differently by different clinicians at the same center.

Clinicians must first differentiate between Long COVID which can be managed with at-home care and relatively benign interventions versus life-threatening illness. Following acute COVID, some patients may develop potentially fatal cases of pulmonary embolism, heart failure, lung fibrosis, stroke as well as severe neurological impairment and mental breakdown $[62,63]$. Acute COVID disrupts the body's immune and endocrine systems, which can exacerbate comorbid conditions and patients may have to deal with this long after the acute COVID resolves. In this connection, it must be noted that not all illnesses that develop in a patient following acute COVID are necessarily Long COVID [62].

When treating a patient who may have Long COVID, it is important to take a thorough history, including medications, and prioritize patient symptoms. A social history may be important in this context, as extended periods of isolation, anxiety about the pandemic, economic hardships, and bereavement are important considerations that can worsen a patient's mental health. Biometric readings, such as oxygen saturation, are crucial. Red flag symptoms include chest pain and profound dyspnea. It may be appropriate in some patients to assess exertional desaturation by having them walk around the room for about 40 steps and testing oxygen saturation levels. Oxygen saturation should be $\geq 95 \%$ and not drop by more than $3 \%$ during exertion [62].

Depending on the patient, it may be helpful to have blood tests. Patients with cardiac comorbidities or cardiac symptoms may benefit from an electrocardiograph. Patients with chest pain or respiratory symptoms may benefit from a chest radiograph [62]. Some patients may require at-home breathing exercises, although more systematic and formal rehabilitation services may be necessary for severe cases.

Patients whose primary symptom is fatigue and whose other symptoms are relatively mild or manageable may respond well to outpatient treatment. Nonsteroidal anti-inflammatory drugs or acetaminophen may be appropriate for myalgia, headache, pain control, and fever. Patients with known comorbidities, such as diabetes, may be counseled to be strict in maintaining their usual care. Mental health symptoms may be managed in some cases by encouraging patients to get peer support, sometimes available online, or re-establish social connections, but formal psychological counseling may be necessary in other cases [62].

The role of physical exercise and workouts in the treatment of Long COVID is uncertain and controversial. Since many Long COVID patients experience profound and overwhelming fatigue along with limited aerobic capacity, some clinicians have discouraged physical exertion while others think it is worthwhile to rebuild physical condition. If patients decide to exercise, they may be well advised to take things slowly, pace themselves, and stop a workout if symptoms worsen [62].

Long COVID can be challenging for family and general practice physicians to manage, and it has been recommended that difficult, complex, or severe cases of Long COVID and patients who do not exhibit clear improvement over time be referred to specialists in Long COVID, who admittedly are few [38]. Despite advances in acute COVID treatments and the vaccine, it is not clear how Long COVID will continue to infect the general population, whether people can be infected after vaccination, and if the virus will mutate like influenza viruses. As case numbers continue to mount, healthcare services will have to deal with increasingly large numbers of Long COVID cases [39]. See Table 3 (Ref. [64]).

\subsection{Guidelines}

The National Institute for Health and Care Excellence (NICE) has published a "living guideline" to address the emerging condition of Long COVID [15]. NICE guidelines recommend for suspected cases of Long COVID blood tests (full blood count; clotting profile; renal, liver, and thyroid functions; C-reactive protein) as well as tests of cardiac and pulmonary function [15]. However, gastrointestinal, neurological, endocrine, and other problems may also occur in Long COVID [15]. In some cases, an appropriate diagnostic assessment of Long COVID requires a multidisciplinary approach and may require multidisciplinary treatment and rehabilitation [15]. More guidance and awareness are needed as patients with Long COVID report difficulty in obtaining an accurate diagnosis, getting appropriate care, and even finding healthcare professionals who show them professional empathy [36].

Of course, any guidelines right now would be at best "living documents", subject to revision with lived clinical experience [3]. Establishing consistent Long COVID terminology and setting forth a comprehensive clinical description of the condition is crucial, which may be premature at this point. The guidelines should note the apparent relapsing-remitting nature of the syndrome, at least in some cases. Furthermore, the current emphasis on social support and cognitive behavioral therapy sometimes observed may undermine the fact that Long COVID appears to be a distinct pathological condition (or more than one), rather than the byproduct of mental stress [3].

\section{Discussion}

Healthcare professionals are only gradually coming to the realization that Long COVID may represent the next major wave of the pandemic. With few studies of the condition and little solid scientific evidence from randomized clinical trials, healthcare professionals, public health experts, and scientists are grappling with a novel syndrome primarily through information from anecdotal reporting. Certainly, serious study is needed to better understand the nature, mechanisms, symptoms, and trajectory of Long COVID. Registries, observational studies, clinical trials, case control studies, and case reports are urgently needed. The use of data collection apps and other novel methods may also be helpful, but do not replace the more traditional role of clinical study.

Frontline healthcare professionals must be empathetic to patients who describe post-infection symptoms of COVID and take these reports seriously. While we currently do not have reliable diagnostic or assessment tools for Long COVID, patients should not feel dismissed when they seek help. Clinical 
TA B L E 3. A good rapport is important in navigating the terrain of this new Long COVID syndrome and there still remain many unknowns [64].

$\begin{array}{ll}\text { Clinical approach } & \text { Relevance } \\ \text { Listen to the patient } & \begin{array}{l}\text { Many Long COVID patients feel marginalized, Patient self-reports are helping to define } \\ \text { discounted, and even disbelieved, which exacer- this disease, so patients should be given the } \\ \text { bates their distress and isolation }\end{array}\end{array}$

Document symptoms

Long COVID is an emerging disease and more The more that is known about Long data are urgently needed COVID, the better treatments can be

Investigate low oxygen levels, heart These Long COVID symptoms can be particu- It may be important in some Long COVID rate, and dyspnea in particular larly dangerous patients to triage symptoms and refer certain patients for more urgent care

Do not confuse Long COVID with Physical symptoms are likely not secondary to Anxiety and depression should be treated, ordinary anxiety anxiety or depression in Long COVID if appropriate, but not everyone who feels anxious after recovering from COVID necessarily has Long COVID

Monitor symptomatic fluctuations Long COVID may exhibit relapsing-remitting Better knowledge of the syndrome may patterns or changes of symptoms improve care

Be alert for "red flags" Some Long COVID symptoms may be severe Such patients should be receive emergency and even potentially life threatening, such as care at once pulmonary embolism, stroke, myocarditis, and others

Educate patients and their families A clinical team member should be able to discuss Patients should be reassured that these Long COVID with patients and their families are real symptoms and given appropriate symptomatic support

Patients should be alerted to what symptoms to look for and that relapsing/remitting patterns may occur

Be aware of patients who do not Improvement with Long COVID may be slow Patients who do not improve for many improve but patients should over the course of weeks and weeks/months should be referred to Long months exhibit improvement

COVID specialists or COVID clinics

Treat symptoms as appropriate

Long COVID may cause headache, myalgia, Symptomatic treatment is appropriate and respiratory problems, nausea, gastrointestinal may help restore function symptoms and so on

Encourage patients to rest and offer Long COVID is frequently associated with Patients should be reassured that this is a strategies to help manage fatigue profound fatigue, which may be one of its more real symptom and that they may need lots enduring syndromes of extra rest to manage

definitions, better terminology, and diagnostic metrics are likewise urgently needed. Public health, payers, and government authorities must be aware that some COVID patients will need ongoing medical care, possibly rehabilitation, mental health services, or other forms of support long after the acute infection resolves [65].

Research is urgently needed to better define Long COVID, in particular phenotypes of the syndrome, risk factors, prognostic markers, natural history, diagnostic tools, and treatment options [15]. Long COVID symptoms may occur weeks and months after the acute infection and recovered COVID patients who report with suspicious symptoms should be carefully evaluated [52].

In an era that emphasizes the value of patient-centric medicine, Long COVID may be a condition in which patients were central to its identification, definition, and description. It has been shown that the early 2020 definition of COVID recovery needs to change from merely "a negative COVID test" to freedom from COVID-related symptoms.

There are several limitations to this article. It is a narrative review of a growing body of literature. Terminology issues have hampered the study and evaluation of Long COVID. The initial reports and interest in Long COVID emerged as a result of self-reported patient experiences, which can be informative but must always be reviewed with academic caution.

\section{Conclusions}

Long COVID is a real and, for some patients, debilitating syndrome that occurs after viral clearance. Research is needed to better understand this pathology and how to manage it appropriately. With millions of people around the world having survived acute COVID, it is likely that the Long COVID syndrome will represent a major new wave of challenge to an already beleaguered healthcare system. Diagnostic challenges in Long COVID along with difficulties in treating it should 
not deter clinicians from recognizing this as a real syndrome and treating patients who report these prolonged or perplexing syndromes with professional empathy and respect.

\section{AUTHOR CONTRIBUTIONS}

JVP had the initial idea. JALQ carried out most of the research, and prepared the first draft of the manuscript. All the authors have contributed to ameliorate the quality of the paper. All named authors meet the International Committee of Medical Journal Editors (ICMJE) criteria for authorship for this article, take responsibility for the integrity of the work as a whole, and have given their approval for this version to be published.

\section{ETHICS APPROVAL AND CONSENT TO PARTICIPATE}

This article is based on previously conducted studies and does not contain any studies with human participants or animals performed by any of the authors.

\section{ACKNOWLEDGMENT}

The authors thank all the patients with Long COVID who have diffused on internet clinical information about themselves, and their feelings.

\section{FUNDING}

This manuscript is funded by NEMA Research Group and Paolo Procacci Foundation. No rapid service fee was received by the journal for the publication of this article.

\section{CONFLICT OF INTEREST}

JVP and GV are members of the Editorial Board of this journal. The other authors do not have any potential conflict of interest to declare.

\section{REFERENCES}

[1] Guillot X, Ribera A, Gasque P. Chikungunya-induced arthritis in reunion island: a long-term observational follow-up study showing frequently persistent joint symptoms, some cases of persistent chikungunya immunoglobulin $\mathrm{m}$ positivity, and no anticyclic citrullinated peptide seroconversion after 13 years. The Journal of Infectious Diseases. 2020; 222: 1740-1744.

[2] Clark DV, Kibuuka H, Millard M, Wakabi S, Lukwago L, Taylor A, et al. Long-term sequelae after Ebola virus disease in Bundibugyo, Uganda: a retrospective cohort study. The Lancet Infectious Diseases. 2015; 15 : 905-912.

[3] Gorna R, MacDermott N, Rayner C, O'Hara M, Evans S, Agyen L, et al. Long COVID guidelines need to reflect lived experience. The Lancet. 2020; 397: 455-457.

[4] Ashton J. Long COVID — what doesn't kill you may not make you stronger. Journal of the Royal Society of Medicine. 2020; 113: 466-467.

[5] Mahase E. COVID-19: what do we know about "Long COVID"? British Medical Journal. 2020; 370: m2815.

[6] Callard F, Perego E. How and why patients made Long COVID. Social Science \& Medicine. 2021; 268: 113426.

[7] Nath A. Long-haul COVID. Neurology. 2020; 95: 559-560.
[8] Body Politic. Body Politic COVID-19 Support Group Newsletter. 2021. Available at: https://www.wearebodypolitic.com/bodytype/ 2021/1/5/the-preprint-youve-been-waiting-for (Accessed: 08 February 2021).

[9] Pergolizzi JV, LeQuang JA, Taylor R, Wollmuth C, Nalamachue M, Varrassi G, et al. Four pandemics: lessons learned, lessons lost. Signa Vitae. 2021; 17: 1-5.

[10] Barber C. The problem of 'Long Haul' COVID. 2020. Available at: https://ww. scientificamerican.com/article/theproblem-of-long-haul-COVID/ (Accessed: 05 February 2021).

[11] Mendelson M, Nel J, Blumberg L, Madhi SA, Dryden M, Stevens W, et al. Long-COVID: an evolving problem with an extensive impact. South African Medical Journal. 2020; 111: 10-12.

[12] Mahase E. Long COVID could be four different syndromes, review suggests. British Medical Journal. 2020; 371: m3981.

[13] Baig AM. Chronic COVID syndrome: need for an appropriate medical terminology for Long-COVID and COVID Long-Haulers. Journal of Medical Virology. 2021; 93: 2555-2556.

[14] Dani M, Dirksen A, Taraborrelli P, Torocastro M, Panagopoulos D, Sutton $\mathrm{R}$, et al. Autonomic dysfunction in 'Long COVID': rationale, physiology and management strategies. Clinical Medicine. 2020; 21: e63-e67.

[15] Sivan M, Taylor S. NICE guideline on Long COVID. British Medical Journal. 2020; 371: m4938.

[16] Datta SD, Talwar A, Lee JT. A proposed framework and timeline of the spectrum of disease due to SARS-CoV-2 infection. The Journal of the American Medical Association. 2020; 324: 2251-2252.

[17] Soriano V, Ganado-Pinilla P, Sánchez-Santos M, Barreiro P. Unveiling Long COVID-19 disease. AIDS Reviews. 2020; 22: 227-228.

[18] Erku DA, Belachew SA, Abrha S, Sinnollareddy M, Thomas J, Steadman $\mathrm{KJ}$, et al. When fear and misinformation go viral: Pharmacists' role in deterring medication misinformation during the 'infodemic' surrounding COVID-19. Research in Social and Administrative Pharmacy. 2021; 17 : 1954-1963.

[19] Carfì A, Bernabei R, Landi F. Persistent symptoms in patients after acute COVID-19. The Journal of the American Medical Association. 2020; 324: 603-605.

[20] Tenforde MW, Kim SS, Lindsell CJ, Billig Rose E, Shapiro NI, et al. Symptom duration and risk factors for delayed return to usual health among outpatients with COVID-19 in a multistate health care systems network-United States, March-June 2020. Morbidity and Mortality Weekly Report. 2020; 69: 993-998.

[21] Huang C, Huang L, Wang Y, Li X, Ren L, Gu X, et al. 6-month consequences of COVID-19 in patients discharged from hospital: a cohort study. The Lancet. 2021; 397: 220-232.

[22] Petersen MS, Kristiansen MF, Hanusson KD, Danielsen ME, Á Steig B, Gaini S, et al. Long COVID in the Faroe Islands-a longitudinal study among non-hospitalized patients. Clinical Infectious Diseases. 2020: ciaa1792. (in press)

[23] Moreno-Pérez O, Merino E, Leon-Ramirez J, Andres M, Ramos JM, Arenas-Jiménez J, et al. Post-acute COVID-19 syndrome. Incidence and risk factors: a Mediterranean cohort study. Journal of Infection. 2021; 82: 378-383.

[24] Brodin P. Immune determinants of COVID-19 disease presentation and severity. Nature Medicine. 2021; 27: 28-33.

[25] Yelin D, Margalit I, Yahav D, Runold M, Bruchfeld J. Long COVID19-it's not over until? Clinical Microbiology and Infection. 2021; 27: 506-508. (in press)

[26] Amenta EM, Spallone A, Rodriguez-Barradas MC, El Sahly HM, Atmar RL, Kulkarni PA. Postacute COVID-19: an overview and approach to classification. Open Forum Infectious Diseases. 2020; 7: ofaa509.

[27] Vaira LA, Salzano G, Fois AG, Piombino P, De Riu G. Potential pathogenesis of ageusia and anosmia in COVID-19 patients. International Forum of Allergy \& Rhinology. 2020; 10: 1103-1104.

[28] Mandal S, Barnett J, Brill SE, Brown JS, Denneny EK, Hare SS, et al. 'Long-COVID': a cross-sectional study of persisting symptoms, biomarker and imaging abnormalities following hospitalisation for COVID-19. Thorax. 2020; 76: 396-398.

[29] Vaira LA, Salzano G, Deiana G, De Riu G. Anosmia and ageusia: common findings in COVID-19 patients. Laryngoscope. 2020; 130: 1787. 
[30] Suzuki M, Saito K, Min W, Vladau C, Toida K, Itoh H, et al. Identification of viruses in patients with postviral olfactory dysfunction. The Laryngoscope. 2007; 117: 272-277.

[31] Lechien JR, Chiesa-Estomba CM, De Siati DR, Horoi M, Le Bon $\mathrm{SD}$, Rodriguez A, et al. Olfactory and gustatory dysfunctions as a clinical presentation of mild-to-moderate forms of the coronavirus disease (COVID-19): a multicenter European study. European Archives of OtoRhino-Laryngology. 2020; 277: 2251-2261.

[32] Mao L, Jin H, Wang M, Hu Y, Chen S, He Q, et al. Neurological manifestations of hospitalized patients with COVID-19 in Wuhan, China: a retrospective case series study. JAMA Neurology. 2020; 77: 683-690.

[33] Brann DH, Tsukahara T, Weinreb C, Lipovsek M, Van den Berge K, Gong $\mathrm{B}$, et al. Non-neuronal expression of SARS-CoV-2 entry genes in the olfactory system suggests mechanisms underlying COVID-19-associated anosmia. Science Advances. 2020; 6: eabc5801.

[34] Papa A, Salzano AM, Di Dato MT, Varrassi G. Images in practice: painful cutaneous vasculitis in a SARS-CoV-2 IgG-positive child. Pain and Therapy. 2020; 9: 805-807.

[35] McMahon DE, Gallman AE, Hruza GJ, Rosenbach M, Lipoff JB, Desai SR, et al. Long COVID in the skin: a registry analysis of COVID-19 dermatological duration. The Lancet Infectious Diseases. 2021; 21: 313 314.

[36] Kingstone T, Taylor AK, O’Donnell CA, Atherton H, Blane DN, ChewGraham CA. Finding the 'right' GP: a qualitative study of the experiences of people with long-COVID. BJGP Open. 2020; 4: bjgpopen20X101143.

[37] Raudenská J, Steinerová V, Javůrková A, Urits I, Kaye AD, Viswanath $\mathrm{O}$, et al. Occupational burnout syndrome and post-traumatic stress among healthcare professionals during the novel coronavirus disease 2019 (COVID-19) pandemic. Best Practice \& Research Clinical Anaesthesiology. 2020; 34: 553-560.

[38] Nabavi N. Long COVID: how to define it and how to manage it. British Medical Journal. 2020; 370: m3489.

[39] Higgins V, Sohaei D, Diamandis EP, Prassas I. COVID-19: from an acute to chronic disease? Potential long-term health consequences. Critical Reviews in Clinical Laboratory Sciences. 2020: 1-23.

[40] Lee M, Kang J, Jeong YJ. Risk factors for post-intensive care syndrome: a systematic review and meta-analysis. Australian Critical Care. 2020; 33: 287-294.

[41] Bienvenu OJ, Friedman LA, Colantuoni E, Dinglas VD, Sepulveda KA, Mendez-Tellez $\mathrm{P}$, et al. Psychiatric symptoms after acute respiratory distress syndrome: a 5-year longitudinal study. Intensive Care Medicine. 2018; 44: 38-47.

[42] Myers EA, Smith DA, Allen SR, Kaplan LJ. Post-ICU syndrome: rescuing the undiagnosed. Journal of the American Academy of Physician Assistants 2016; 29: 34-37.

[43] Meeting the challenge of Long COVID. Nature Medicine. 2020; 26: 1803.

[44] Pergolizzi J, Raffa R, Fleischer C, Taylor R, LeQuang J. Chikungunya: basics for clinicians and their patients. Journal of General and Family Medicine. 2017; 1: 1-12.

[45] Moldofsky H, Patcai J. Chronic widespread musculoskeletal pain, fatigue, depression and disordered sleep in chronic post-SARS syndrome; a casecontrolled study. BMC Neurology. 2011; 11: 37.

[46] Puntmann VO, Carerj ML, Wieters I, Fahim M, Arendt C, Hoffmann J, et al. Outcomes of cardiovascular magnetic resonance imaging in patients recently recovered from coronavirus disease 2019 (COVID-19). JAMA Cardiology. 2020; 5: 1265-1273.

[47] Klok FA, Kruip MJHA, van der Meer NJM, Arbous MS, Gommers DAMPJ, Kant KM, et al. Incidence of thrombotic complications in critically ill ICU patients with COVID-19. Thrombosis Research. 2020; 191: 145-147.

[48] Patell R, Bogue T, Koshy A, Bindal P, Merrill M, Aird WC, et al.
Postdischarge thrombosis and hemorrhage in patients with COVID-19. Blood. 2020; 136: 1342-1346.

[49] Sharma R, Sardar S, Mohammad Arshad A, Ata F, Zara S, Munir W. A patient with asymptomatic SARS-CoV-2 infection who presented 86 days later with COVID-19 pneumonia possibly due to reinfection with SARSCoV-2. American Journal of Case Reports. 2020; 21: e927154.

[50] Centers for Disease Control and Prevention. Duration of isolation and precautions for adults with COVID-19. 2020. Available at: https: //www.cdc.gov/coronavirus/2019-ncov/hcp/durationisolation.html (Accessed: 30 January 2021).

[51] Halpin S, O'Connor R, Sivan M. Long COVID and chronic COVID syndromes. Journal of Medical Virology. 2020; 93: 1242-1243.

[52] Lokugamage AU, Bowen MA, Blair J. Long COVID: doctors must assess and investigate patients properly. British Medical Journal. 2020; 371: m4583.

[53] Franceschi C, Salvioli S, Garagnani P, de Eguileor M, Monti D, Capri M. Immunobiography and the heterogeneity of immune responses in the elderly: a focus on inflammaging and trained immunity. Frontiers in Immunology. 2017; 8: 982.

[54] Franceschi C, Garagnani P, Parini P, Giuliani C, Santoro A. Inflammaging: a new immune-metabolic viewpoint for age-related diseases. Nature Reviews. Endocrinology. 2018; 14: 576-590.

[55] Bektas A, Schurman SH, Franceschi C, Ferrucci L. A public health perspective of aging: do hyperinflammatory syndromes such as COVID19, SARS, ARDS, cytokine storm syndrome, and post-ICU syndrome accelerate short- and long-term inflammaging? Immunity \& Ageing. 2020; 17: 23.

[56] De Martinis M, Franceschi C, Monti D, Ginaldi L. Inflamm-ageing and lifelong antigenic load as major determinants of ageing rate and longevity. FEBS Letters. 2005; 579: 2035-2039.

[57] Franceschi C, Garagnani P, Vitale G, Capri M, Salvioli S. Inflammaging and 'Garb-aging' trends in endocrinology and metabolism: Transmission Electron Microscopy. 2017; 28: 199-212.

[58] Furman D, Campisi J, Verdin E, Carrera-Bastos P, Targ S, Franceschi C, et al. Chronic inflammation in the etiology of disease across the life span. Nature Medicine. 2019; 25: 1822-1832.

[59] Rekatsina M, Paladini A, Piroli A, Zis P, Pergolizzi JV, Varrassi G. Pathophysiology and therapeutic perspectives of oxidative stress and neurodegenerative diseases: a narrative review. Advances in Therapy. 2020; 37: 113-139.

[60] Ludvigsson JF. Case report and systematic review suggest that children may experience similar long-term effects to adults after clinical COVID-19. Acta Paediatrica. 2020; 110: 914-921.

[61] Ladds E, Rushforth A, Wieringa S, Taylor S, Rayner C, Husain L, et al. Persistent symptoms after COVID-19: qualitative study of 114 "Long COVID” patients and draft quality principles for services. BMC Health Services Research. 2020; 20: 1144

[62] Greenhalgh T, Knight M. Long COVID: a primer for family physicians. American Academy of Family Physicians. 2020; 102: 716-717.

[63] Pergolizzi JV Jr, Raffa RB, Varrassi G, Magnusson P, LeQuang JA, Paladini A, et al. Potential neurological manifestations of COVID-19: a narrative review. Postgraduate Medical Journal. 2021; 1-11.

[64] Atherton H, Briggs T, Chew-Graham C. Long COVID and the importance of the doctor-patient relationship. British Journal of General Practice. 2021; 71: 54-55.

[65] The Lancet. Facing up to Long COVID. The Lancet. 2020; 396: 1861

How to cite this article: Pergolizzi $\mathrm{J}$ V, LeQuang $\mathrm{J}$ A, Magnusson P, Myrcik D, Varrassi G. It's not over till it's over: a narrative review of Long COVID. Signa Vitae. 2021;17(3):2130. doi:10.22514/sv.2021.067. 